\title{
Resiniferatoxin Induces Paradoxical Changes in Thermal and Mechanical Sensitivities in Rats: Mechanism of Action
}

\author{
Hui-Lin Pan, ${ }^{1,2}$ Ghous M. Khan, ${ }^{1}$ Kevin D. Alloway, ${ }^{2}$ and Shao-Rui Chen ${ }^{1}$ \\ Departments of ${ }^{1}$ Anesthesiology and ${ }^{2}$ Neuroscience and Anatomy, The Pennsylvania State University College of Medicine, The Milton S. Hershey Medical \\ Center, Hershey, Pennsylvania 17033-0850
}

\begin{abstract}
Resiniferatoxin (RTX), an ultrapotent analog of capsaicin, has been used as a tool to study the role of capsaicin-sensitive C fibers in pain. Recently, we found that RTX diminished the thermal sensitivity but unexpectedly increased the sensitivity to tactile stimulation in adult rats. In this study, we explored the potential mechanisms involved in RTX-induced changes in somatosensory function. An intraperitoneal injection of $200 \mu \mathrm{g} / \mathrm{kg}$ RTX, but not its vehicle, rapidly produced an increase in the paw withdrawal latency to a heat stimulus. Also, profound tactile allodynia developed in all the RTX-treated rats in 3 weeks. This paradoxical change in thermal and mechanical sensitivities lasted for at least 6 weeks. Electron microscopic examination of the sciatic nerve revealed a loss of unmyelinated fibers and extensive ultrastructural damage of myelinated fibers in RTX-treated rats. Immunofluorescence labeling showed a diminished vanilloid receptor 1 immunoreactivity in dorsal root ganglia neurons and the spinal dorsal horn of RTX-treated rats. Furthermore, two transganglionic tracers, horseradish peroxidase conjugates of cholera toxin B subunit (CTB) and isolectin- $\mathrm{B}_{4}$ of Bandeiraea simplicifolia (IB ${ }_{4}$ ), were injected into the opposite sides of the sciatic nerve to trace myelinated and unmyelinated afferent terminations, respectively, in the spinal dorsal horn. In RTX-treated rats, $\mathrm{IB}_{4}$-labeled terminals in the dorsal horn were significantly reduced, and CTB-labeled terminals appeared to sprout into lamina II of the spinal dorsal horn. Thus, this study demonstrates that systemic RTX diminishes the thermal pain sensitivity by depletion of unmyelinated afferent neurons. The delayed tactile allodynia induced by RTX is likely attributable to damage to myelinated afferent fibers and their abnormal sprouting in lamina II of the spinal dorsal horn. These data provide new insights into the potential mechanisms of postherpetic neuralgia.
\end{abstract}

Key words: neuropathic pain; postherpetic neuralgia; capsaicin; VR1 receptor; TRPV channel; spinal cord dorsal horn; axonal sprouting

\section{Introduction}

Capsaicin, the pungent ingredient of hot pepper, excites smalldiameter primary sensory neurons involved in nociception. The administration of capsaicin depletes most unmyelinated afferent fibers in neonatal rats (Jancso et al., 1977; Nagy and van der Kooy, 1983). Resiniferatoxin (RTX), which is an ultrapotent analog of capsaicin, binds to the capsaicin receptor vanilloid receptor 1 [VR1; also known as transient receptor potential ion channel (TRPV1)] expressed on primary sensory neurons that mediate thermal pain perception (Szallasi and Blumberg, 1989; Szallasi et al., 1989; Caterina et al., 2000). Because systemic RTX treatment can deplete capsaicin-sensitive $\mathrm{C}$ fibers in adult rats, it has been used as a pharmacological tool to study the role of nociceptive $\mathrm{C}$ fibers in the development of neuropathic pain in rodent models (Hao et al., 1996; Ossipov et al., 1999; Khan et al., 2002). Recently, we used RTX to determine the role of capsaicin-sensitive afferent fibers in the development of diabetic neuropathic pain in rats (Khan et al., 2002). During the course of that study, we unexpectedly found that systemic RTX diminished the thermal sensitivity but caused an increased sensitivity to tactile stimulation in adult

Received 0ct. 28, 2002; revised Dec. 12, 2002; accepted Jan. 13, 2003.

This work was supported by National Institutes of Health (NIH) Grants GM64830, NS41178, and HL04199. H.-L.P was a recipient of an Independent Scientist Award supported by NIH during the course of this study. We are grateful to Pamela Myers for her secretarial assistance and to Roland Myers for his technical support.

Correspondence should be addressed to Dr. Hui-Lin Pan, Department of Anesthesiology, H187, Pennsylvania State University College of Medicine, 500 University Drive, Hershey, PA 17033. E-mail: hpan@psu.edu.

Copyright $\odot 2003$ Society for Neuroscience $\quad 0270-6474 / 03 / 232911-09 \$ 15.00 / 0$ rats. The effect of this neurotoxin on the distinct changes in somatosensory function has not been reported previously. In the present study, we determined the possible sites and mechanisms of the action of RTX on altered thermal and mechanical sensitivities in rats.

The VR1 receptors located on primary afferent fibers and neurons are important for the transmission of acute pain elicited by heat and capsaicin (Caterina et al., 2000). A characteristic of chronic neuropathic pain is abnormal responsiveness to cutaneous stimulation. Painful sensation can be evoked by an innocuous stimulus such as touch (tactile allodynia) in patients with neuropathic pain (Gracely et al., 1992). The existing rodent models of neuropathic pain display increased thermal and mechanical sensitivities after peripheral nerve injury. However, the paradoxical changes in thermal and mechanical sensitivities are present in patients with small-fiber neuropathies, in particular postherpetic neuralgia (Baron and Saguer, 1993; Rowbotham and Fields, 1996). The mechanisms underlying this interesting clinical phenomenon are not fully known. Both peripheral and central mechanisms contribute to the development of neuropathic pain syndromes (Devor and Wall, 1981; Woolf et al., 1992; Matzner and Devor, 1994; Kohama et al., 2000). Peripheral-nerve injury elicits a number of electrophysiological and molecular changes, including abnormal sensitization of nociceptors and development of ectopic discharge activity in damaged nerves (Koltzenburg et al., 1994; Matzner and Devor, 1994; Khan et al., 2002). Thus, we examined histologically the changes of myelinated and unmyelinated fibers in the sciatic nerve of RTX-treated rats. The effect of 
RTX on VR1 receptors expressed on primary sensory neurons was also determined.

Furthermore, damage to primary afferent nerves can trigger secondary changes in sensory processing in the spinal dorsal horn (Woolf et al., 1992, 1995; Koerber et al., 1994). The spinal dorsal horn has distinct layers that receive and process inputs from different sensory receptors (Rivero-Melian and Grant, 1990; Woodbury et al., 2000). Some central terminals of myelinated afferent fibers are found in the inner lamina II but never synapse in the outer lamina II, an area that receives input exclusively from unmyelinated C fibers (Woolf et al., 1992, 1995; Koerber et al., 1994; Woodbury et al., 2000). Peripheral-nerve transection induces collateral sprouting of myelinated afferents from deeper laminas into the lamina II of the spinal cord, which normally receives inputs exclusively from unmyelinated nociceptive afferents (Woolf et al., 1992, 1995; Koerber et al., 1994). Such a structural reorganization has been considered as an important anatomical basis for the development of allodynia after peripheral-nerve injury (Woolf et al., 1992; Mannion et al., 1996). Therefore, in this study we also used two transganglionic tracers to determine how RTX affected the laminar specificity of inputs from myelinated and unmyelinated afferent terminals in the spinal dorsal horn.

\section{Materials and Methods}

Animals. Experiments were conducted on male Sprague Dawley (Harlan, Indianapolis, IN) rats weighing between 280 and $300 \mathrm{gm}$. The procedures and protocols were approved by the Animal Care and Use Committee of the Pennsylvania State University College of Medicine. Each rat in the RTX group $(n=10)$ received a single intraperitoneal injection of RTX $\left(200 \mu \mathrm{g} / \mathrm{kg}\right.$; Sigma, St. Louis, MO) under halothane $\left(2 \%\right.$ in $\left.\mathrm{O}_{2}\right)$ anesthesia. RTX was dissolved in a mixture of $10 \%$ Tween 80 and $10 \%$ ethanol in normal saline (Khan et al., 2002). Each rat in the control group $(n=6)$ received a corresponding vehicle injection. Before RTX or vehicle treatment, the baseline sensitivity of each rat to mechanical and thermal stimulation was measured. After injection, the rats in both groups were tested every 2-3 d for 8 weeks.

Sensitivity to mechanical stimulation. All rats were tested to determine the withdrawal threshold of the hindpaw in response to von Frey filaments. Behavioral testing was conducted between 8:30 and 11:30 A.M. Rats were individually placed in suspended chambers on a mesh floor. After an acclimation period of $30 \mathrm{~min}$, a series of calibrated von Frey filaments (Stoelting, Wood Dale, IL) were applied perpendicularly to the plantar surface of both hindpaws with sufficient force to bend the filament for 6 sec. Brisk withdrawal or paw flinching was considered a positive response. In the absence of a response, the filament of the next greater force was applied. After a response, the filament of the next lower force was applied. The tactile stimulus producing a 50\% likelihood of withdrawal response was calculated by using the "up-down" method, as described previously (Chaplan et al., 1994; Chen and Pan, 2002). The test was repeated two to three times in each rat, and the mean value was calculated.

Sensitivity to thermal stimulation. Sensitivity to noxious heat stimulation was determined by placing each rat in an individual Plexiglas enclosure on a transparent glass surface maintained at $30^{\circ} \mathrm{C}$. After a $30 \mathrm{~min}$ acclimation period, the heat-emitting projector lamp of a thermal testing apparatus (IITC Inc./Life Sciences Instruments, Woodland Hills, CA) was activated after focusing the beam directly onto the plantar surface of the hindpaw. A built-in digital timer was used to record the paw withdrawal latency. The mean value of the withdrawal latency on two to three consecutive trials was calculated. A cutoff of $30 \mathrm{sec}$ was used to avoid potential tissue damage (Khan et al., 2002).

Immunofluorescence labeling of VR1 receptors. Immunofluorescence labeling of VR1 receptors in dorsal root ganglia (DRG) neurons and the spinal dorsal horn was performed on three vehicle- and four RTX-treated rats to determine the effects of RTX on VR1 receptors located on primary afferent neurons and central terminals. Each rat was deeply anesthetized with sodium pentobarbital (60 mg/kg, i.p.) and perfused intracardially with $200 \mathrm{ml}$ ice-cold normal saline containing $1000 \mathrm{U}$ of heparin followed by $500 \mathrm{ml}$ paraformaldehyde in $0.1 \mathrm{M}$ PBS, pH 7.4, and $200 \mathrm{ml} \mathrm{10 \%}$ sucrose in $0.1 \mathrm{M}$ PBS, pH 7.4. The lumbar segment of the spinal cord and DRG neurons were quickly removed and postfixed for $2 \mathrm{hr}$ in the same fixative solution and cryoprotected in $30 \%$ sucrose in $0.1 \mathrm{M}$ PBS for $48 \mathrm{hr}$ at $4^{\circ} \mathrm{C}$. The sections were cut at $25 \mu \mathrm{m}$ and collected freely floating in 0.1 M PBS. For VR1 receptor immunofluorescence labeling, the sections were rinsed in $0.1 \mathrm{M}$ PBS and blocked in 4\% normal goat serum in PBS for $1 \mathrm{hr}$. The sections were then incubated with the primary antibody (rabbit anti-VR1 N terminal, dilution 1:1000; Neuromics, Minneapolis, MN) diluted in PBS containing 2\% normal goat serum, $0.3 \%$ Triton X-100, and $0.05 \%$ Tween 20 for $2 \mathrm{hr}$ at room temperature and overnight at $4^{\circ} \mathrm{C}$. Subsequently, sections were rinsed in PBS and incubated with the secondary antibody (Alexa Fluor-488 conjugated to goat anti-rabbit IgG, dilution: $5 \mu \mathrm{g} / \mathrm{ml}$; Molecular Probes, Eugene, OR). A fluorescence microscope was used to view the sections, and areas of interest were photodocumented.

Electron microscopic examination of the sciatic nerve. Electron microscopy was used to assess the ultrastructural changes of myelinated and unmyelinated fibers in RTX- and vehicle-treated rats. At the end of behavioral testing, two vehicle- and three RTX-treated rats were anesthetized with pentobarbital (50 mg/kg, i.p.), and the right sciatic nerve was surgically removed and fixed in $1 \%$ paraformaldehyde (Fisher Scientific, Pittsburgh, PA) and 2.5\% glutaraldehyde (Sigma-Aldrich) solution. After $24 \mathrm{hr}$, tissues were transferred into sodium acetate buffer solution. The tissue was then postfixed in $1 \%$ osmium tetroxide for $2 \mathrm{hr}$, dehydrated in a series of graded ethanol solutions, and treated with propylene oxide. The tissue sections were dipped in a series of resin and propylene oxide mixtures in varying proportions and then infiltrated with $100 \%$ resin. Finally, the sections were embedded in $100 \%$ freshly prepared resin and incubated in an oven, and ultrathin sections were cut using a Sorvall (Newtown, CT) MT-2B ultramicrotome, poststained with $2 \%$ aqueous uranyl acetate and Reynold's lead citrate, and mounted on mesh grids. Photomicrographs were taken on a transmission electron microscope (Philips transmission electron microscope model 400; Philips, Acht, The Netherlands). To quantify the number of myelinated and unmyelinated axons in the sciatic nerve, 10 high-power fields $(\times 4600)$ were randomly selected from each sciatic-nerve section from two vehicle- and two RTXtreated rats. The total number of myelinated and unmyelinated axons in each high-power field was counted.

Tracing of afferent projections to the dorsal horn. Two vehicle- and three RTX-treated rats were injected with two transganglionic tracers, horseradish peroxidase conjugates of cholera toxin B-subunit (CTB) and isolectin- $\mathrm{B}_{4}$ of Bandeiraea simplicifolia $\left(\mathrm{IB}_{4}\right)$. $\mathrm{CTB}$ and $\mathrm{IB}_{4}$ have been used to map the central projections of cutaneous myelinated and unmyelinated primary afferents, respectively, in the spinal dorsal horn of rats (Woodbury et al., 2000). At the end of behavioral testing, CTB (1\%, List Biological Laboratories, Campbell, CA) and $\mathrm{IB}_{4}$ (1\%; Sigma) were injected into opposite sciatic nerves to determine the pattern of labeled afferent terminals in the dorsal horn of RTX- and vehicle-treated rats. The sciatic nerve on each side was exposed at the midthigh level after the rats were anesthetized by an intramuscular injection of a mixture of xylazine $(10 \mathrm{mg} / \mathrm{kg})$ and ketamine $(60 \mathrm{mg} / \mathrm{kg})$. Small volumes $(2.5-3 \mu \mathrm{l})$ of $\mathrm{CTB}$ and $\mathrm{IB}_{4}$ tracers were loaded into Hamilton microsyringes and injected into the sciatic nerves on opposite sides of the body in the same animal to allow bilateral comparisons of the resulting labeling patterns in the dorsal horn (Woodbury et al., 2000).

After allowing 3-5 d for transganglionic axoplasmic transport of both tracers, each rat was deeply anesthetized with sodium pentobarbital (60 $\mathrm{mg} / \mathrm{kg}$, i.p.) and transcardially perfused with $50 \mathrm{ml}$ heparinized normal saline. The rat was then perfused with $500 \mathrm{ml} 1.25 \%$ glutaraldehyde and $1 \%$ paraformaldehyde in $0.1 \mathrm{M}$ PBS, pH 7.4, for $30 \mathrm{~min}$, followed by 500 $\mathrm{ml} 10 \%$ sucrose in $0.1 \mathrm{M}$ PBS for $30 \mathrm{~min}$. The spinal cord was quickly removed and collected in ice-cold $10 \%$ sucrose PBS and stored at $4^{\circ} \mathrm{C}$. The lumbar spinal cord (L4-L6) was dissected out, and serial coronal sections (40 $\mu \mathrm{m}$ in thickness) were processed for tetramethylbenzidine (TMB) staining as described previously (Mesulam, 1978). Briefly, the sections were rinsed in water before a prereaction soak in freshly prepared solution A ( $100 \mathrm{mg}$ of sodium nitroferricyanide in $0.2 \mathrm{M}$ sodium 
acetate) and solution B (5 mg of TMB in $2.5 \mathrm{ml}$ ethanol) for $20 \mathrm{~min}$ at room temperature. Solution A was added to solution B just before the prereaction soak. Free-floating sections were incubated with $0.3 \%$ hydrogen peroxide for $20 \mathrm{~min}$ at room temperature, stabilized in sodium ferricyanide and $0.2 \mathrm{M}$ sodium acetate buffer for $20 \mathrm{~min}$ at $4^{\circ} \mathrm{C}$, and washed in $0.1 \mathrm{M}$ PBS. Finally, sections were mounted on gelatin-subbed slides, and then counterstained with $0.1 \%$ neutral red, dehydrated, and defatted in alcohol and xylene and coverslipped.

Reconstructions and analysis of afferent terminals in the dorsal horn. An Olympus BH-2 light microscope (Olympus Optical, Tokyo, Japan) was used to view the nerve terminals impregnated with the tracers. A microscopic reconstruction system (MD-3; Accustage, St. Paul, MN) was used to plot the location of the CTB- and $\mathrm{IB}_{4}$-labeled terminals in the dorsal horn of lumbar spinal sections. Every third section mounted on a slide was arbitrarily selected for plotting. Anatomic outlines of the section and the gray matter were first plotted with a $10 \times$ objective. The CTB- and $\mathrm{IB}_{4}$-labeled terminals were then plotted, using red and blue colors, respectively, with a $40 \times$ objective. Digital reconstructions of the topography of labeled terminals were saved and stored on a computer disk for subsequent retrieval. The area occupied by CTB- and $\mathrm{IB}_{4}$-labeled fibers was quantified from the digital reconstructions by using a JAVA-based software program (Alloway et al., 2000). This program subdivided each plotted section into an array of $10 \mu \mathrm{m}^{2}$ bins. The number of CTB (red) and $\mathrm{IB}_{4}$ (blue) labeled bins were counted and summed across all plotted sections, and the mean number of red- and blue-labeled bins in each section was calculated. A Coolsnap HQ CCD digital camera (BioVision Technologies, Inc., Exton, PA) was used to obtain photomicrographs of the labeled terminals in the spinal dorsal horn.

Statistical analysis. Data are presented as means \pm SEM. The effect of RTX on the paw withdrawal threshold and latency was analyzed using repeated-measures ANOVA followed by Dunnett's post hoc test. The effect of RTX on CTB- and $\mathrm{IB}_{4}$-labeled afferent terminals in the spinal dorsal horn was determined using Student's $t$ test. A $p$ value of $<0.05$ was considered statistically significant.

\section{Results}

In this study, all 10 rats treated with RTX survived. After recovering from anesthesia, animals injected with RTX exhibited immediate behavioral reactions, including hyperexcitability and restlessness. However, these effects were transient, and they gradually subsided within 2-3 hr. Otherwise, the RTX-treated rats appeared normal throughout the experiment.

\section{Thermal sensitivity}

The effect of RTX on thermal sensitivity was examined by testing the paw withdrawal latency in response to noxious heat stimulation. Figure 1 shows the time course of changes in thermal sensitivity in 6 vehicle- and 10 RTX-treated rats. Before treatment, the paw withdrawal latency elicited by the radiant heat stimulus was $7.20 \pm 0.68 \mathrm{sec}$ in control rats and $7.81 \pm 0.51 \mathrm{sec}$ in RTX-treated rats. In the RTX group, the withdrawal latency of both hindpaws tested was significantly increased and nearly reached the cutoff of $30 \mathrm{sec}$ within $2 \mathrm{~d}$ after RTX administration (Fig. 1). No significant changes in thermal sensitivity were observed in the vehicletreated group during the 8 week period of the experiment (Fig. 1).

\section{Mechanical sensitivity}

Mechanical sensitivity was determined by measuring the paw withdrawal threshold in response to application of von Frey filaments. Figure 2 illustrates the time course of changes in mechanical sensitivity of rats that received vehicle or RTX injections. Before treatment, the paw withdrawal thresholds were similar in both vehicle- (18.59 $\pm 1.89 \mathrm{gm} ; n=6)$ and RTX-treated (19.01 \pm 2.22 gm; $n=10)$ rats. However, two weeks after RTX treatment the paw withdrawal threshold in response to mechanical stimulation began to decrease (Fig. 2). By the third week, a large reduc- tion in the withdrawal threshold was apparent after RTX treatment. Furthermore, immediately after stimulus-induced withdrawal, RTX-treated rats often licked the paw that was stimulated by the von Frey filaments. This decreased withdrawal threshold occurred in both hindpaws tested and remained sustained for the duration of the experiment. By contrast, no significant changes in the paw withdrawal thresholds were observed in any of the six vehicle-treated rats during the 8 week period of the experiment (Fig. 2).

\section{Ultrastructural changes of nerve fibers in the sciatic nerve}

Electron microscopic examination of the sciatic-nerve sections revealed the characteristic appearance of myelinated and unmyelinated fibers in vehicle-treated rats. As shown in Figure $3 A$, myelinated fibers were larger in size, approximately circular in shape, and were bounded by a compact wall of the myelin sheath. The unmyelinated fibers were much smaller, roughly oval in shape, and arranged in well defined layers or clusters that were interspersed among the myelinated fibers. However, in sciaticnerve sections from RTX-treated rats, there was a substantial depletion of unmyelinated fibers (Fig. 3B-D). Also, considerable damage to myelinated fibers was evident in the sciatic nerve of RTX-treated rats. Ultrastructural changes of myelinated fibers included axonal swelling, altered myelination, and the appearance of abnormal Schwann cells. Swelling of myelinated axons also caused a marked reduction in the interstitial space (Fig. $3 B$ $D)$. Compared with the circular appearance of myelinated fibers in vehicle-treated rats, the damaged myelinated fibers appeared distorted and had a more elongated or wedged-shaped configuration in RTX-treated rats (Fig. 3B-D). However, typical signs of Wallerian degeneration (such as the breakdown of the myelin sheath and fragments of axons) were not observed in the sciatic nerve of RTX-treated rats. The total number of myelinated axons per high-power field $(\times 4600)$ in the sciatic nerve of vehicle- and RTX-treated rats was $42.3 \pm 2.1$ and $39.8 \pm 2.3(p>0.05)$, respectively. Unmyelinated axons in the sciatic nerve of RTXtreated rats were substantially depleted compared with those in vehicle-treated rats $(6.3 \pm 1.2$ vs $64.4 \pm 3.1$ per high-power field; $p<0.05)$.

\section{VR1 receptor immunoreactivity in DRG neurons and spinal dorsal horn}

To examine the effect of RTX on VR1 receptor-expressing primary afferent neurons, VR1 receptor-immunofluorescent labeling of primary afferent neurons was conducted in the DRG from vehicle- and RTX-treated rats. VR1 receptor immunoreactivity was present in small and medium-sized DRG neurons in vehicletreated rats (Fig. 4). In contrast, the VR1 receptor immunoreactivity was substantially depleted in DRG neurons from RTXtreated rats (Fig. 4).

To determine the effect of RTX on the VR1 receptors on the central terminals of primary afferents, we also examined VR1 receptor immunoreactivity in the spinal dorsal horn. Figure 4 shows VR1 receptor immunoreactivity in the spinal dorsal horn of a vehicle- and an RTX-treated rat. In vehicle-treated rats, dense VR1 receptor immunoreactivity was seen both at the dorsal root entry zone and in laminas I and II. By comparison, VR1 receptor immunoreactivity was completely abolished in the superficial dorsal horn in RTX-treated rats (Fig. 4). These findings indicate that RTX treatment depletes VR1 receptor-expressing afferent neurons and their central terminals in the spinal dorsal horn. 


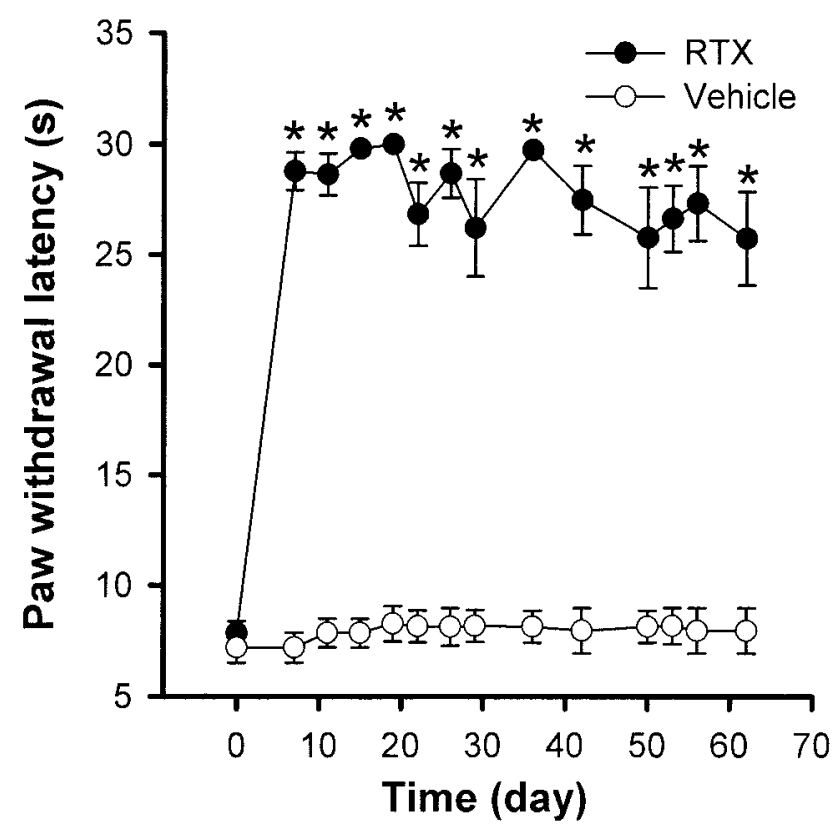

Figure 1. Time course of the paw withdrawal latency to a noxious heat stimulus in 6 vehicleand 10 RTX-treated rats. ${ }^{*} p<0.05$ compared with the pretreatment control. The paw withdrawal latency was determined by a radiant heat stimulus.

\section{Transganglionic labeling of afferent terminals in the dorsal horn}

Transganglionic transport of $\mathrm{CTB}$ and $\mathrm{IB}_{4}$ on opposite sides of the vehicle-treated rat resulted in reciprocal, nonoverlapping patterns of labeling in the dorsal horn, indicating that these two markers labeled distinct subsets of primary afferent nerves. Preferential labeling of myelinated fibers by CTB allowed us to determine whether RTX produced any significant changes in the innervation pattern of these afferent fibers in the dorsal horn. As shown in Figure 5, all CTB-labeled terminals in the dorsal horn were found ipsilateral to the injection site in both vehicle- and RTX-treated rats. In the vehicle-treated rats, widespread CTBlabeled terminals were observed in all dorsal horn laminas except the lamina II area (Fig. 5A,B). In both vehicle and RTX groups, CTB labeling was quite dense in the medial part of laminas III to $\mathrm{V}$, but it was relatively sparse along the ventrolateral parts of laminas IV and V. However, in contrast to the control group, spinal cord sections from the RTX-treated rats contained scattered CTB labeling in spinal lamina II (Fig. 5C). As shown in Figure $5 C$, some CTB-labeled terminals appeared to enter lamina II from lamina III, and the dorsal horn area corresponding to lamina II was considerably smaller in RTX-treated rats. These findings suggest that myelinated afferent fibers have sprouted into lamina II in RTX-treated rats. Despite the difference in the amount of CTB-labeled afferent terminals that appeared in the superficial dorsal horn, we did not detect a significant difference in the total spatial extent of CTB-labeled terminals in the dorsal horn area between vehicle- and RTX-treated groups (Fig. 6). The slight decrease in CTB labeling in the whole dorsal horn is not inconsistent with the presence of apparent sprouting in lamina II of RTX-treated animals.

Mapping the $\mathrm{IB}_{4}$-labeled terminals in the spinal dorsal horn allowed us to make a quantitative assessment of the effect of RTX on the central terminals of unmyelinated afferents. Figure $5 \mathrm{~A}$ illustrates representative reconstructions that show the location of $\mathrm{IB}_{4}$-labeled terminals in the spinal dorsal horn of a vehicle- and

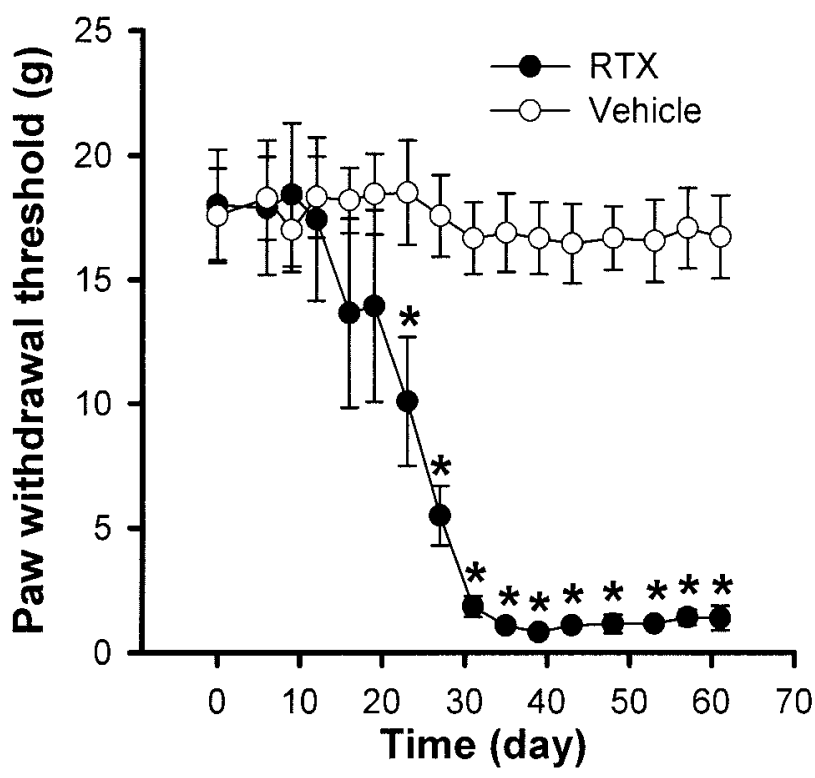

Figure 2. Time course of the development of tactile allodynia in 10 rats treated with RTX and the mechanical withdrawal threshold in 6 vehicle-treated rats. ${ }^{*} p<0.05$ compared with the pretreatment control. The paw withdrawal thresholds were determined using von Frey filaments.

an RTX-treated rat. In vehicle-treated rats, the dense $\mathrm{IB}_{4}$-positive terminals occupied lamina I and the outer zone of lamina II in the region left vacant by $\mathrm{CTB}$ across the mediolateral plane of the dorsal horn (Fig. $5 A, B$ ). The $\mathrm{IB}_{4}$-labeled terminals appeared as a planar sheet along the contour of the superficial dorsal horn and were densest in the lateral part of this sheet. However, the dorsoventral location of the sheet varied across the mediolateral plane. As the planar sheet extended to the lateral aspect, a relatively fine layer of $\mathrm{IB}_{4}$-labeled terminals occupied a more superficial position and closely followed the contour of the dorsal horn. In contrast to the prominent mediolateral spread of the $\mathrm{IB}_{4}$-labeled terminals as a planar sheet in vehicle-treated rats, $\mathrm{IB}_{4}$-labeled terminals in RTX-treated rats appeared either as faint patches or as a very thin sheet of labeling (Fig. $5 A, B$ ). In many dorsal horn sections from the RTX group, $\mathrm{IB}_{4}$-positive terminals were almost absent. Also, in RTX-treated rats, $\mathrm{IB}_{4}$-labeled terminals in the superficial dorsal horn occupied significantly less space than the space occupied by these terminals in vehicle-treated rats $(p<$ 0.05 ) (Fig. 6). The reduction in $\mathrm{IB}_{4}$-labeled afferent terminals by RTX is consistent with the depletion of VR1 receptor immunoreactivity in the superficial dorsal horn of RTX-treated rats.

\section{Discussion}

The present study demonstrates that a single intraperitoneal injection of RTX substantially reduced thermal sensitivity, but produced profound and persistent tactile allodynia in adult rats. The mechanisms underlying these paradoxical effects on thermal and mechanical sensitivity were investigated. We found that RTX depleted VR1 receptor immunoreactivity in DRG neurons and the central terminals of afferent fibers. Also, there was a significant reduction in $\mathrm{IB}_{4}$-labeled unmyelinated afferent terminals in the superficial dorsal horn of RTX-treated rats. Ultrastructural examination of the sciatic nerve revealed that RTX caused a loss of unmyelinated fibers and produced detectable levels of damage to the myelinated nerve fibers. Furthermore, CTB-labeled myelinated afferent terminals appeared to expand into lamina II in the spinal dorsal horn of RTX-treated rats. Thus, this study indicates 

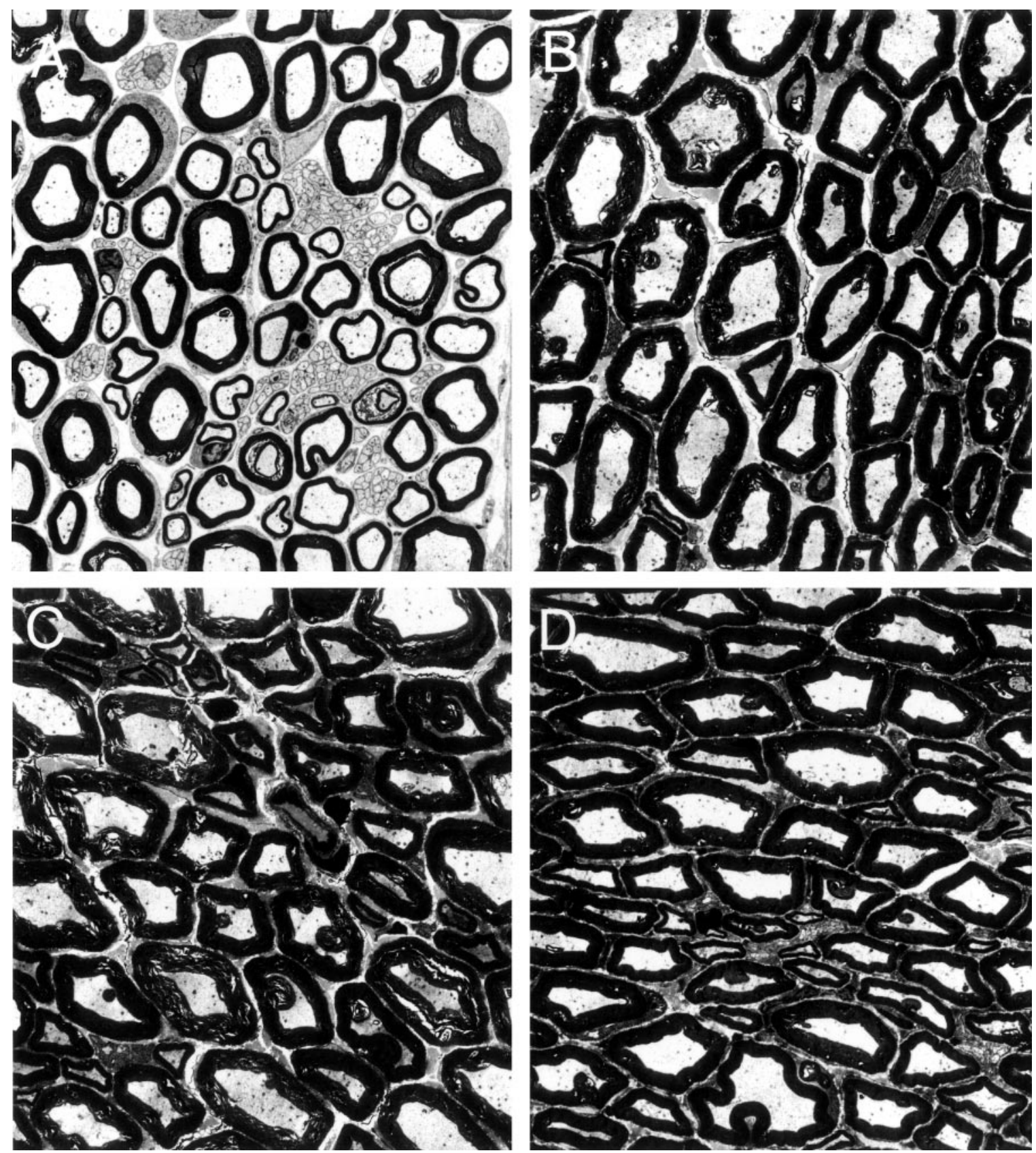

Figure 3. Representative electron photomicrographs showing ultrastructural changes of myelinated fibers in the sciatic nerve in a vehicle $(A)$ - and an RTX ( $B-D)$-treated rats. Note that the ultrastructural changes induced by RTX include the loss of unmyelinated fibers and noticeable swelling of the myelinated fibers. Magnification, $\times 4439$.

that systemic RTX diminishes thermal pain perception by depleting primary sensory neurons that express VR1 receptors. The RTX-induced tactile allodynia is probably attributable to damage to both myelinated afferent nerves and their abnormal sprouting in lamina II of the spinal dorsal horn.

RTX elicits an immediate nociceptive response characterized by irritation, pungency, and burning pain attributable to a brief excitation of primary sensory neurons (Szallasi and Blumberg, 1989). This transient action is followed by a prolonged desensitization and degeneration of C-fiber afferent neurons (Szallasi and Blumberg, 1989; Ossipov et al., 1999; Khan et al., 2002). We observed that a single RTX injection produced a rapid and significant increase in the paw withdrawal latency to noxious heat stimulation. Thus, capsaicin-sensitive unmyelinated afferent fi- bers may become desensitized or may degenerate after RTX treatment. The permanent effect of RTX on unmyelinated fibers was indicated by a significant reduction in $\mathrm{IB}_{4}$-labeled afferent terminals in the spinal dorsal horn and the loss of unmyelinated fibers in the sciatic nerve of RTX-treated rats. Using VR1 receptor knock-out mice, it has been shown that VR1 receptors are essential for acute pain caused by noxious heat and capsaicin injection (Caterina et al., 2000). Consistent with these findings, we found that VR1 receptor immunoreactivity in the DRG neurons was depleted in RTX-treated rats. Also, the transganglionic tracer $\mathrm{IB}_{4}$ was used in this study to map the central projection of unmyelinated cutaneous afferents in the spinal cord. RTX treatment produced a marked reduction of both $\mathrm{IB}_{4}$-labeled afferent terminals and VR1 receptor immunoreactivity in the superficial dorsal 
horn. Because $\mathrm{IB}_{4}$ labels both capsaicinsensitive and non-capsaicin-sensitive unmyelinated fibers (Petruska et al., 2000), our data suggest that most $\mathrm{IB}_{4}$ labeled afferent terminals in the superficial dorsal horn are capsaicin-sensitive afferent fibers expressing VR1 receptors. The long-lasting effect of RTX on diminished thermal sensitivity suggests that capsaicin-sensitive afferent neurons have a limited capacity to regenerate (Scadding, 1980). Thus, this study provides strong evidence that RTX causes a long-lasting diminished thermal sensitivity through depletion of unmyelinated afferent nerves and neurons.

The profound and persistent tactile allodynia in RTX-treated rats was unexpected. To our knowledge, this delayed effect of RTX has not been found previously. To determine the potential sites of the action of RTX, we examined the effect of RTX on the peripheral nerve and the topographical projection of afferent terminals in the spinal dorsal horn. Our analyses showed that RTX induced ultrastructural changes indicative of damage to the myelinated fibers in the sciatic nerve. This finding suggests that the effects of RTX were not confined only to the unmyelinated $\mathrm{C}$ fibers. In fact, it has been shown recently that VR1 receptors are also located on $\sim 30 \%$ of myelinated A-fiber afferent neurons ( $\mathrm{Ma}, 2002$ ). Because myelinated afferent fibers play an important role in allodynia development after peripheralnerve injury (Hao et al., 1996; Ossipov et al., 1999), the tactile allodynia in RTX-treated rats could be explained, at least in part, by the abnormal activity from damaged myelinated afferent fibers. However, significant changes in the mechanosensitivity did not occur until 3 weeks after RTX treatment. If RTX-induced damage to myelinated fibers is a direct consequence of VR1 receptor activation, then changes in the mechanical withdrawal threshold might be expected to occur sooner after RTX treatment. But a previous study did not observe ultrastructural changes in the myelinated DRG neurons 2 weeks after RTX treatment (Szallasi et al., 1989). Because the ultrastructural change of myelinated fibers was observed in most myelinated fibers in the sciatic nerve of RTX-treated rats, the delayed allodynic action of RTX cannot be fully explained by the activation of VR1 receptors expressed in some myelinated afferent fibers. Instead, the delayed allodynia and structural damage of myelinated afferent fibers induced by RTX could be caused by mechanisms unrelated to the stimulation of VR1 receptors. In this regard, both capsaicin and RTX have been reported to inhibit the NADPH-plasma membrane electron transport system, and this may induce apoptosis in the affected neurons (Macho et al., 1998). Also, the ultrastructural changes of myelinated fibers may be secondary to the surrounding unmyelinated fiber degeneration caused by RTX. Previous studies have shown that injured myelinated afferent nerves develop ectopic activities that could alter and amplify the sensory input so that an innocuous stimulus could be interpreted as being painful (Gracely et al., 1992; Koltzenburg et al., 1994; Matzner and Devor, 1994; Michaelis et al., 1995). Thus, these ectopic discharges from damaged myelinated afferents could induce and
Control
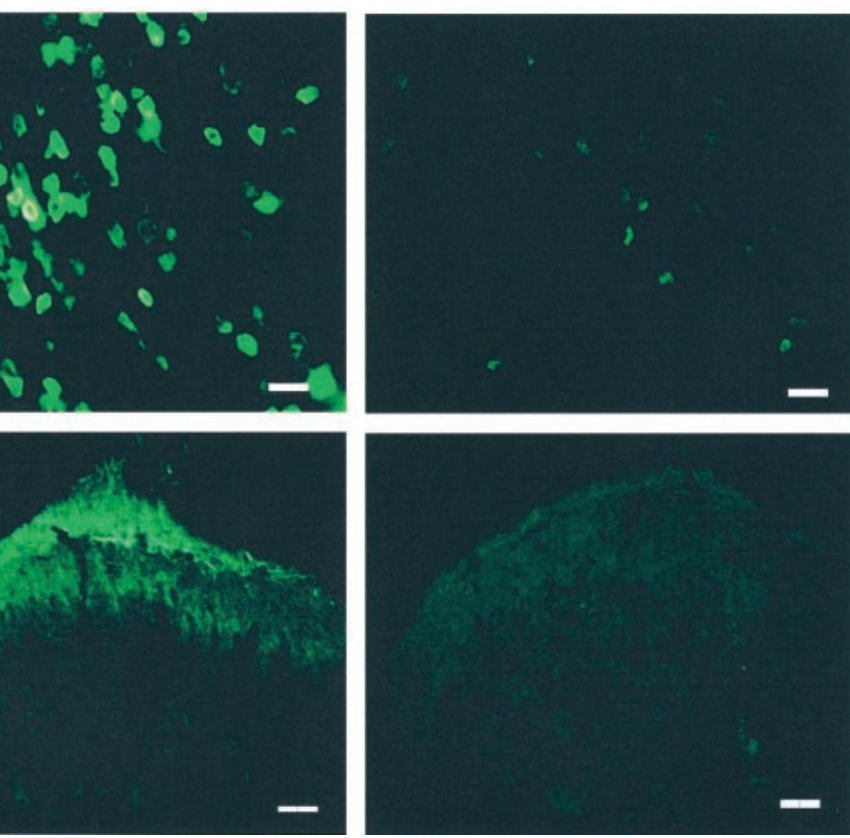

\section{RTX}

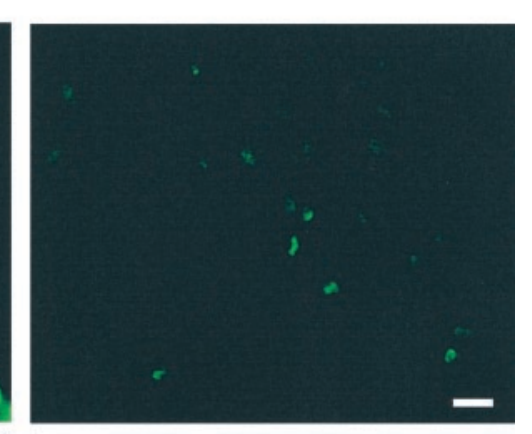

(n)

Figure 4. VR1 receptor immunoreactivity in lumbar DRG and spinal dorsal horn in a vehicle- and an RTX-treated rat. Densely stained VR1 receptor immunoreactivity is present in the DRG and superficial dorsal horn in

maintain a state of hypersensitivity of spinal dorsal horn neurons, thereby resulting in allodynia. Furthermore, the development of transneuronal degeneration and loss of dorsal horn inhibitory neurons after the depletion of unmyelinated afferent fibers may constitute an additional mechanism for the gradual increase in the mechanical sensitivity in RTX-treated rats.

Alternatively, the delayed allodynic effect of RTX might be attributable to the time required for primary afferent nerves to regenerate and terminate on incorrect targets in the spinal dorsal horn (Andrew and Greenspan, 1999). The terminal arbors of primary afferents are topographically organized in the normal spinal dorsal horn. Using CTB as a tracer for myelinated fibers, it has been shown that myelinated fibers sprout into lamina II within 2 weeks after peripheral axotomy or crush (Woolf et al., 1992). To determine whether RTX triggers sprouting of myelinated afferents in lamina II of the spinal cord, we used two transganglionic tracers, $\mathrm{IB}_{4}$ and $\mathrm{CTB}$, to preferentially label the central projections of unmyelinated and myelinated cutaneous afferents, respectively (Robertson and Arvidsson, 1985; Rivero-Melian and Grant, 1990; Wang et al., 1994; Woodbury et al., 2000). We found that CTB-labeled terminals of myelinated afferent terminals expanded into the spinal lamina II in RTX- but not vehicle-treated rats. This finding is consistent with previous studies showing that transection of the sciatic nerve causes myelinated afferents to sprout from lamina III into lamina II (Woolf et al., 1992, 1995). Lamina II normally receives mostly C-fiber afferent inputs, and the expansion of myelinated afferent fibers into lamina II could alter the peripheral input to produce a painful response to touch (Woolf et al., 1992, 1995). The mechanism by which myelinated fibers sprout into lamina II is not known. Both the creation of vacant synaptic space within lamina II after C-fiber degeneration and the regeneration of damaged myelinated fibers may induce sprouting of myelinated fibers into this region in RTX-treated 

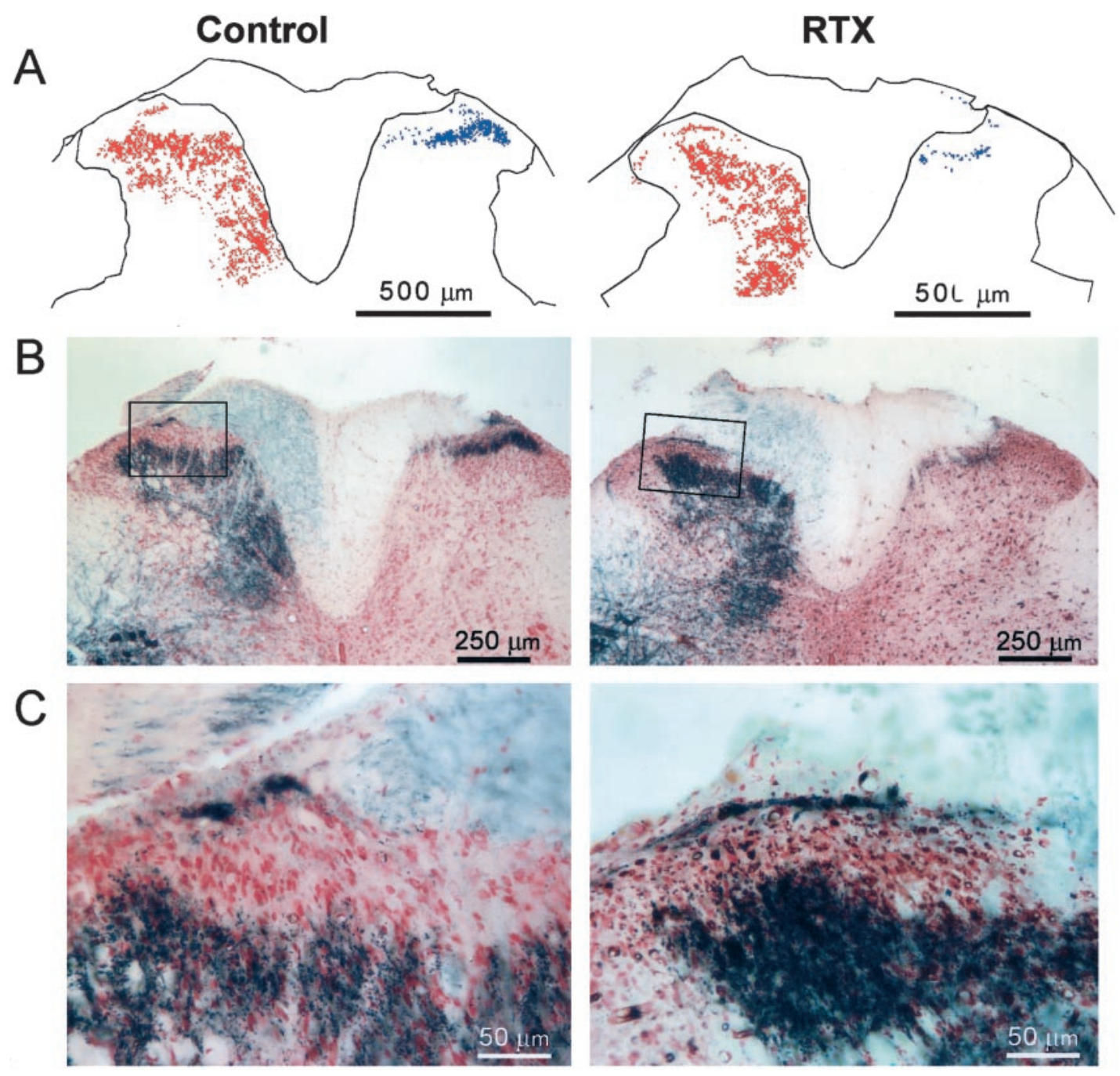

\section{RTX}
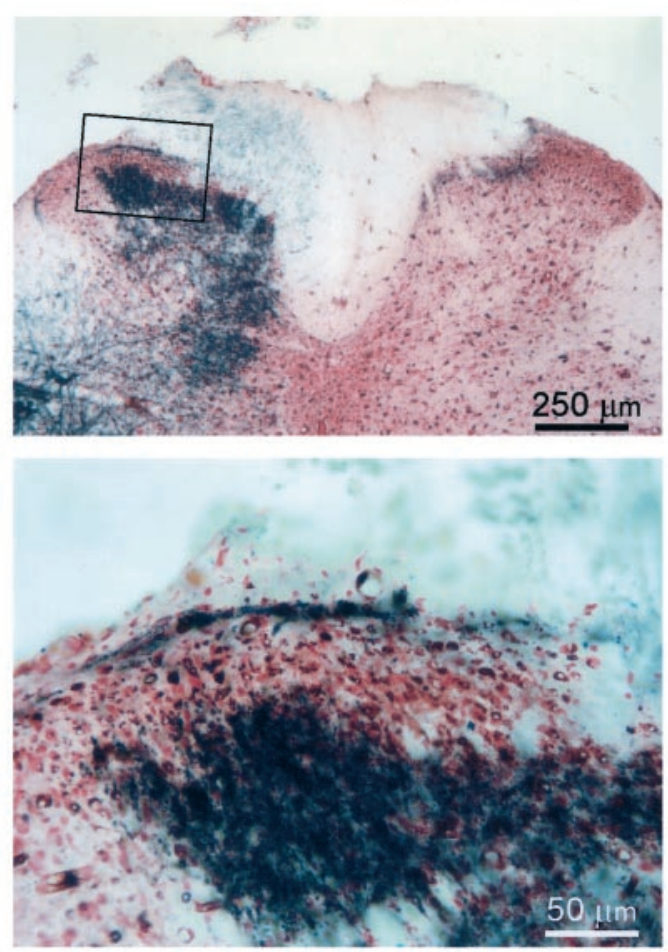

Figure 5. Photomicrographs showing reconstructed plots $(A)$ and the original staining photomicrographs $(B)$ of $C T B$ - and $\mathrm{IB}_{4}$-labeled afferent terminals in the spinal dorsal horn of a vehicle control and an RTX-treated rat. In $A$ and $B$, the left side of the dorsal horn contains $\left(T B\right.$-labeled terminals and the right side contains $\mathrm{IB}_{4}$-labeled afferent terminals. $C$, Magnification of the inset in $B$. Note that the scattered (TB-labeled terminals are present in lamina II of the RTX-treated rat (C).

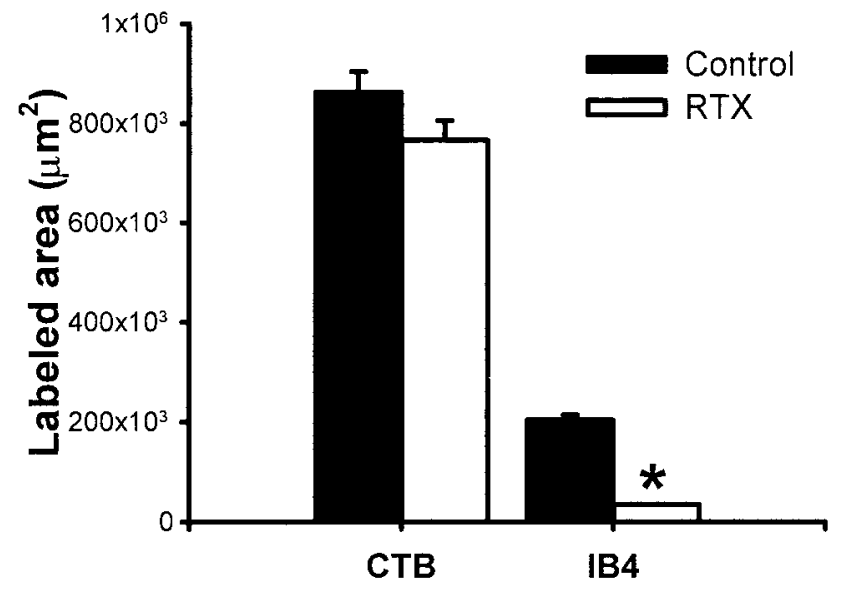

Figure 6. Mean amount of $\mathrm{CTB}$ - and $\mathrm{IB}_{4}$-labeled areas in the dorsal horn of lumbar spinal sections in two vehicle- ( 26 sections) and three RTX-treated ( 40 sections) rats. The mean area was calculated from reconstructed sections as shown in Figure $5 A$. Data are presented as means \pm SEM. ${ }^{*} p<0.05$ compared with the control in the $\mathrm{IB}_{4}$ group (Student's $t$ test). rats (Woolf et al., 1992, 1995; Mannion et al., 1996, 1998). Unmyelinated afferent terminals undergo transganglionic degeneration resulting from the depletion of VR1 receptor-expressing DRG neurons by RTX. After the atrophy of unmyelinated C fibers, the presence of vacant synaptic sites within the superficial dorsal horn has been suggested as an important factor for eliciting sprouting from neighboring intact myelinated terminals (Woolf et al., 1992, 1995). Given the substantial reduction of unmyelinated afferent terminals produced by RTX in lamina II, this could promote axonal sprouting of myelinated afferent fibers. However, C-fiber damage or depletion may not be essential for myelinated afferent sprouting in the spinal lamina II (Mannion et al., 1998). Nonetheless, the noticeable extension of myelinated afferent fibers into lamina II may represent a form of axonal sprouting that contributes to the development of persistent tactile allodynia in RTX-treated rats. To our knowledge, this is the first study showing that functional changes in mechanical sensitivity correlate with anatomical plasticity at the spinal cord level. This important finding extends our current knowledge of spinal mechanisms of chronic neuropathic pain.

The apparent expansion of myelinated fibers into lamina II 
was not as pronounced as reported in previous studies (Woolf et al., 1992, 1995). One possible reason for this difference in degree might be the difference in the cause of the nerve damage (i.e., surgical nerve transection versus chemically induced nerve injury by RTX). Also, it has been shown that peripheral axotomy can cause a marked increase in the uptake and transganglionic transport of CTB by a subpopulation of small-sized DRG neurons (Tong et al., 1999). Thus, it has been suggested that at least part of the sprouting of CTB-labeled fibers in lamina II after nerve transection could be attributable to phenotypic changes resulting in small-sized DRG neurons with unmyelinated axons becoming CTB-positive (Tong et al., 1999). Such a phenotypic switch caused by nerve transection suggests that axonal sprouting in the dorsal horn may be less pronounced than was originally assumed. In support of this view, a recent study has shown only a limited sprouting of CTB-labeled myelinated afferents in lamina II after peripheral axotomy in rats (Bao et al., 2002). However, in the present study, RTX depleted most unmyelinated afferent fibers and the small DRG neurons that express VR1 receptors. Thus, it is less likely that sprouting of myelinated afferent terminals in lamina II is attributable to the abnormal uptake of CTB by unmyelinated afferent fibers in the RTX-treated rats. It will be interesting to determine whether nerve injury can cause more sprouting of myelinated terminals in RTX-treated animals than in RTX animals alone.

In summary, we have shown that a single systemic treatment of RTX produces an interesting paradoxical change in thermal and mechanical sensitivities in adult rats. Our data suggest that the diminished thermal sensitivity by RTX is attributable to the depletion of unmyelinated afferent neurons. Furthermore, the delayed persistent tactile allodynia in RTX-treated rats is likely caused by damage of myelinated afferent fibers and their abnormal sprouting into the spinal lamina II. It should be noted that all existing rodent models of neuropathic pain exhibit increased sensitivities to both thermal and mechanical stimuli after peripheral nerve injury. However, in patients with postherpetic neuralgia, profound tactile allodynia and reduced thermal sensitivity often coexist in the affected dermatomes (Baron and Saguer, 1993; Rowbotham and Fields, 1996). Because the distinct behavioral signs displayed in RTX-treated rats are similar to those seen in these patients, RTX-induced tactile allodynia in rats could be used as a unique model for this debilitating neuropathic pain condition. Therefore, data from this study provide important new information for our understanding of the potential mechanisms of postherpetic neuralgia.

\section{References}

Alloway KD, Mutic JJ, Hoffer ZS, Hoover JE (2000) Overlapping corticostriatal projections from the rodent vibrissal representations in primary and secondary somatosensory cortex. J Comp Neurol 428:51-67.

Andrew D, Greenspan JD (1999) Mechanical and heat sensitization of cutaneous nociceptors after peripheral inflammation in the rat. J Neurophysiol 82:2649-2656.

Bao L, Wang HF, Cai HJ, Tong YG, Jin SX, Lu YJ, Grant G, Hokfelt T, Zhang $X$ (2002) Peripheral axotomy induces only very limited sprouting of coarse myelinated afferents into inner lamina II of rat spinal cord. Eur J Neurosci 16:175-185.

Baron R, Saguer M (1993) Postherpetic neuralgia: are C-nociceptors involved in signaling and maintenance of tactile allodynia? Brain 116:1477-1496.

Caterina MJ, Leffler A, Malmberg AB, Martin WJ, Trafton J, Petersen-Zeitz
KR, Koltzenburg M, Basbaum AI, Julius D (2000) Impaired nociception and pain sensation in mice lacking the capsaicin receptor. Science 288:306-313.

Chaplan SR, Bach FW, Pogrel JW, Chung JM, Yaksh TL (1994) Quantitative assessment of tactile allodynia in the rat paw. J Neurosci Methods 53:55-63.

Chen SR, Pan HL (2002) Hypersensitivity of spinothalamic tract neurons associated with diabetic neuropathic pain in rats. J Neurophysiol $87: 2726-2733$

Devor M, Wall PD (1981) Plasticity in the spinal cord sensory map following peripheral nerve injury in rats. J Neurosci 1:679-684.

Gracely RH, Lynch SA, Bennett GJ (1992) Painful neuropathy: altered central processing maintained dynamically by peripheral input. Pain 51:175-194.

Hao JX, Yu W, Xu XJ, Wiesenfeld-Hallin Z (1996) Capsaicin-sensitive afferents mediate chronic cold, but not mechanical, allodynia-like behavior in spinally injured rats. Brain Res 722:177-180.

Jancso G, Kiraly E, Jancso-Gabor A (1977) Pharmacologically induced selective degeneration of chemosensitive primary sensory neurones. Nature 270:741-743.

Khan GM, Chen SR, Pan HL (2002) Role of primary afferent nerves in allodynia caused by diabetic neuropathy in rats. Neuroscience 114:291-299.

Koerber HR, Mirnics K, Brown PB, Mendell LM (1994) Central sprouting and functional plasticity of regenerated primary afferents. J Neurosci 14:3655-3671.

Kohama I, Ishikawa K, Kocsis JD (2000) Synaptic reorganization in the substantia gelatinosa after peripheral nerve neuroma formation: aberrant innervation of lamina II neurons by A $\beta$ afferents. J Neurosci 20:1538-1549.

Koltzenburg M, Torebjork HE, Wahren LK (1994) Nociceptor modulated central sensitization causes mechanical hyperalgesia in acute chemogenic and chronic neuropathic pain. Brain 117:579-591.

Ma QP (2002) Expression of capsaicin receptor (VR1) by myelinated primary afferent neurons in rats. Neurosci Lett 319:87-90.

Macho A, Blazquez MV, Navas P, Munoz E (1998) Induction of apoptosis by vanilloid compounds does not require de novo gene transcription and activator protein 1 activity. Cell Growth Differ 9:277-286.

Mannion RJ, Doubell TP, Coggeshall RE, Woolf CJ (1996) Collateral sprouting of uninjured primary afferent A-fibers into the superficial dorsal horn of the adult rat spinal cord after topical capsaicin treatment to the sciatic nerve. J Neurosci 16:5189-5195.

Mannion RJ, Doubell TP, Gill H, Woolf CJ (1998) Deafferentation is insufficient to induce sprouting of A-fibre central terminals in the rat dorsal horn. J Comp Neurol 393:135-144.

Matzner O, Devor M (1994) Hyperexcitability at sites of nerve injury depends on voltage-sensitive $\mathrm{Na}^{+}$channels. J Neurophysiol 72:349-359.

Mesulam MM (1978) Tetramethyl benzidine for horseradish peroxidase neurohistochemistry: a non-carcinogenic blue reaction product with superior sensitivity for visualizing neural afferents and efferents. J Histochem Cytochem 26:106-117.

Michaelis M, Blenk KH, Janig W, Vogel C (1995) Development of spontaneous activity and mechanosensitivity in axotomized afferent nerve fibers during the first hours after nerve transection in rats. J Neurophysiol 74:1020-1027.

Nagy JI, van der Kooy D (1983) Effects of neonatal capsaicin treatment on nociceptive thresholds in the rat. J Neurosci 3:1145-1150.

Ossipov MH, Bian D, Malan Jr TP, Lai J, Porreca F (1999) Lack of involvement of capsaicin-sensitive primary afferents in nerve-ligation injury induced tactile allodynia in rats. Pain 79:127-133.

Petruska JC, Napaporn J, Johnson RD, Gu JG, Cooper BY (2000) Subclassified acutely dissociated cells of rat DRG: histochemistry and patterns of capsaicin-, proton-, and ATP-activated currents. J Neurophysiol 84:2365-2379.

Rivero-Melian C, Grant G (1990) Distribution of lumbar dorsal root fibers in the lower thoracic and lumbosacral spinal cord of the rat studied with choleragenoid horseradish peroxidase conjugate. J Comp Neurol 299:470-481.

Robertson B, Arvidsson J (1985) Transganglionic transport of wheat germ agglutinin-HRP and choleragenoid-HRP in rat trigeminal primary sensory neurons. Brain Res 348:44-51.

Rowbotham MC, Fields HL (1996) The relationship of pain, allodynia and thermal sensation in post-herpetic neuralgia. Brain 119:347-354. 
Scadding JW (1980) The permanent anatomical effects of neonatal capsaicin on somatosensory nerves. J Anat 131:471-482.

Szallasi A, Blumberg PM (1989) Resiniferatoxin, a phorbol-related diterpene, acts as an ultrapotent analog of capsaicin, the irritant constituent in red pepper. Neuroscience 30:515-520.

Szallasi A, Joo F, Blumberg PM (1989) Duration of desensitization and ultrastructural changes in dorsal root ganglia in rats treated with resiniferatoxin, an ultrapotent capsaicin analog. Brain Res 503:68-72.

Tong YG, Wang HF, Ju G, Grant G, Hokfelt T, Zhang X (1999) Increased uptake and transport of cholera toxin B-subunit in dorsal root ganglion neurons after peripheral axotomy: possible implications for sensory sprouting. J Comp Neurol 404:143-158.
Wang H, Rivero-Melian C, Robertson B, Grant G (1994) Transganglionic transport and binding of the isolectin B4 from Griffonia simplicifolia I in rat primary sensory neurons. Neuroscience 62:539-551.

Woodbury CJ, Ritter AM, Koerber HR (2000) On the problem of lamination in the superficial dorsal horn of mammals: a reappraisal of the substantia gelatinosa in postnatal life. J Comp Neurol 417:88-102.

Woolf CJ, Shortland P, Coggeshall RE (1992) Peripheral nerve injury triggers central sprouting of myelinated afferents. Nature 355:75-78.

Woolf CJ, Shortland P, Reynolds M, Ridings J, Doubell T, Coggeshall RE (1995) Reorganization of central terminals of myelinated primary afferents in the rat dorsal horn following peripheral axotomy. J Comp Neurol 360:121-134. 